\title{
Size-selectivity of predatory reef fish on juvenile prey
}

\author{
Thomas H. Holmes ${ }^{1,2, *}$, Mark I. McCormick ${ }^{1}$ \\ ${ }^{1}$ ARC Centre of Excellence for Coral Reef Studies, and School of Marine and Tropical Biology, James Cook University,
} Townsville, Queensland 4811, Australia

${ }^{2}$ Present address: Marine Science Program, Science Division, Department of Environment and Conservation, Kensington, Western Australia 6151, Australia

\begin{abstract}
For organisms with complex life cycles, the selective nature of predation during high mortality transitional periods is thought to play a large role in determining the community structure of later life stages. Here we investigate the dynamic nature of size selection by predators on coral reef fishes during the transitional early post-settlement period. A series of aquarium-based predator choice trials were used to examine the selective nature of 4 predatory fish species (Pseudochromis fuscus, Thalassoma lunare, Synodus dermatogenys and Cephalopholis microprion) known to be responsible for a majority of predation on early juvenile reef fish. A single species of common damselfish (Pomacentrus amboinensis) was used as a model prey species. Experiments were conducted on both naïve settlement-stage individuals and more 'experienced' early juveniles in order to examine the changing dynamic of the interactions through time. The intensity and direction of size selection was found to differ significantly between predator species during both the settlement and early juvenile stage trials, with some species preferentially removing smaller individuals, while others removed larger individuals or were non-selective. Predator gape size played a limited role across all interactions, while selectivity was not found to differ with changing predator ontogeny. Our results highlight the complexity of predator-prey relationships within multi-species communities and suggest that no specific expression of a phenotypic trait holds a definitive survival advantage during all encounters. Instead, prey survival will in part be determined by the suite of predators present at the location of settlement, and how they interact with the available prey community.
\end{abstract}

KEY WORDS: Predation · Selection · Body size $\cdot$ Gape limitation $\cdot$ Reef fish $\cdot$ Predator ontogeny Resale or republication not permitted without written consent of the publisher

\section{INTRODUCTION}

Understanding the processes underlying population dynamics is one of the key issues confronting ecologists working in complex systems. An intimate knowledge of these processes, and how they interact, is essential before broad scale predictions can be made at community and population levels. Predation is generally thought to play a major role in determining the size of populations and the structure of terrestrial and aquatic communities (Wilbur et al. 1983, Sih et al. 1985, Risbey et al. 2000). However, due to its speed and decisive nature, predation is notoriously difficult to study. The selectivity of predation over ecological time frames (i.e. phenotypic selection) has been widely implicated in determining those character traits that are passed into successive life stages. These selective forces may act on a number of different body and performance characteristics, all of which can influence survival in a variety of situations. Such characteristics include body size (Allen 2008, Sakamoto \& Hanazato 2008), overall condition (Husseman et al. 2003, Penteriani et al. 2008), growth rate (Takasuka et al. 2003, Urban 2007), sensory development (Poling \& Fuiman 1997) and escape speed (Brana 2003). Body size has by far received the most attention in the literature and is generally regarded as one of the major characteristics linked to survival during predatory encounters (Cohen et al. 1993, Wellborn 1994, Sogard 1997, Wang et al. 2007).

One common theory (the 'Bigger-is-Better' hypothesis) suggests that from a prey's perspective, larger size 
at a given life-history stage results in a survival advantage, through lower predation rates (Rice et al. 1993, Congdon et al. 1999, Wang et al. 2007), enhanced competitive abilities and a lower susceptibility to starvation (Stuart-Smith et al. 2007). Thus, as prey size increases, vulnerability to predation is predicted to decrease. In contrast, optimal foraging theory (OFT) predicts that predators preferentially prey on an optimal prey size in order to maximise the net rate of energy intake (MacArthur \& Pianka 1966, Hughes 1980). This theory predicts that both large and small size may convey a survival advantage during a predatory encounter. The characteristics of the prey that are targeted are contingent on the selective preferences of the predator, which tend to be dome-shaped (e.g. Rice et al. 1993).

To understand the influence of body size on the outcome of predatory encounters, the relative sizes of predator and prey need to be considered. Prey selected by a predator depends on the characteristics expressed by both predator and prey, and how these interact (Cohen et al. 1993, Woodward et al. 2005, Urban 2007). For many piscivores, the upper limit of potential prey sizes is set by morphological constraints imposed by mouth width, or gape size (a mechanism known as 'gape-limitation'; Persson et al. 1996, Slaughter \& Jacobson 2008). According to OFT, as predator size increases, the optimal prey size on which to feed should also increase (Hughes 1980). Thus, as predator size increases, individuals are predicted to preferentially select larger prey (Rice et al. 1993, Scharf et al. 2000). This increase in preferred prey size has been attributed to ontogenetic increases in mouth gape, visual acuity, digestive capacity and locomotive performance. As a result of these underlying mechanisms, in relationships involving single species of predator and prey, the size distribution of the predator will determine the size range of prey eaten. The size range of prey available will then determine the nature of size selection.

At the time of settlement to the reef environment, coral reef fishes are subjected to extremely high levels of mortality, with upwards of $60 \%$ of individuals being lost within 48 hours of settlement (Doherty et al. 2004, Almany \& Webster 2006). Much of this mortality is attributed to the actions of small reef-associated predatory fish (Carr \& Hixon 1995, Holbrook \& Schmitt 2002). As a result, there is the potential for predator selection during this period to have a large influence on those traits that are passed on to successive life stages. The gape-limitation imposed on many predatory fish means that the key relationship underlying predator-prey interactions in this system may well be predator mouth width versus the prey body depth (Werner 1974, Werner \& Gilliam 1984, Hill et al. 2005). However, given the diversity of predator morpholo- gies, behaviours and attack modes amongst predatory reef fish, it is reasonable to suggest that different species may exploit the size range of prey in different ways (Holmes \& McCormick 2006). To date, there is little data on the species-specific selectivity of predators within communities for any system. If we are to predict how prey populations may respond to changing predator communities, and vice versa, it is essential that we gain an understanding of how selective patterns differ between predators within a system.

This study examines the nature of size selection by predators on the common Indo-Pacific damselfish Pomacentrus amboinensis, during the early postsettlement period. To investigate the changing dynamics of this process, the selectivity of predators on naïve newly metamorphosed individuals is compared with that of experienced juveniles. Previous research indicates that experience obtained by individuals in the days immediately following settlement increases survival during predatory encounters (McCormick \& Holmes 2006) and hence may also influence selective patterns. Ecological theory suggests that, from a predator's perspective, selective preferences towards a particular prey trait may differ between predator species. However, from a prey's perspective, conventional theory would suggest that a particular expression of that trait provides a generalised survival advantage during all interactions (e.g. bigger-isbetter). We addressed these tenets in a series of aquarium experiments using 4 predator species known to be responsible for a majority of predation on juvenile reef fish on shallow lagoonal reefs throughout the IndoPacific. Specifically, we assessed (1) whether different predator species will differ in their size-selective preferences during predatory interactions with newly metamorphosed and early juvenile prey individuals, (2) whether, in keeping with OFT, preferred prey size increases with increasing predator size, and (3) the role of predator gape size in predator-prey relationships between 4 important predator species and juvenile and settlement-stage prey.

\section{MATERIALS AND METHODS}

Study site and species. This study was conducted at Lizard Island $\left(14^{\circ} 40^{\prime} \mathrm{S}, 145^{\circ} 28^{\prime} \mathrm{E}\right)$, northern Great Barrier Reef (GBR), Australia, during November and December of 2006 and 2007. The flow-through saltwater aquarium system at Lizard Island Research Station was used to conduct the aquarium trials, whilst all fish collections were made from the surrounding shallow lagoonal reefs and sand flats.

The common damselfish Pomacentrus amboinensis was used as the model prey species for all experimental trials. This species is common within coral reef fish 
communities within the Indo-Pacific, particularly in the central GBR. Individuals settle in a wide variety of habitats on the northern GBR, but are found in highest densities associated with small reef patches at the base of shallow reefs. The species has a pelagic larval phase of between 15 and $23 \mathrm{~d}$ and settles at a standard length of between 10.3 and $15.1 \mathrm{~mm}$ (Kerrigan 1996) with its juvenile body plan largely complete (McCormick et al. 2002). Once settled, P. amboinensis is siteattached, making it an ideal species for experimental manipulation. They recruit in substantial numbers at Lizard Island around the new moon during the summer months (October through January) and are easily collected at the time of settlement with light traps (Milicich \& Doherty 1994). This life cycle, body plan and approximate size are common to a large number of damselfish (Pomacentridae) species. Hence, any selective processes found to be operating on $P$. amboinensis in this study may be broadened to a wide range of Pomacentrid species.

Four species of small site-associated reef fish were used as predators during aquarium trials: the brown dottyback Pseudochromis fuscus (Pseudochromidae); the moonwrasse Thalassoma lunare (Labridae); the sand lizardfish Synodus dermatogenys (Synodontidae); and the freckled rockcod Cephalopholis microprion (Serranidae). All species are common on shallow reefs throughout much of the West Pacific and Indian Oceans and are generally thought to be responsible for a majority of predation on newly settled reef fishes in these habitats (Martin 1994, T. H. Holmes \& M. I. McCormick pers. obs.). P. fuscus is a small (max. size $72.4 \mathrm{~mm}$ SL), solitary cryptic pursuit predator commonly found on small coral bommies or along reef edges. T. lunare is a highly active opportunistic predator (max. size $200 \mathrm{~mm} \mathrm{SL}$ ), generally found higher in the water column in haremic groups across a range of reef habitats. $S$. dermatogenys is a cryptic ambush predator (max. size $210 \mathrm{~mm}$ SL) commonly found on sandy substrata immediately adjacent to the reef base or amongst small bommies and coral rubble. C. microprion is another cryptic predator (max. size $210 \mathrm{~mm}$ $\mathrm{SL}$ ), generally found in caves or beneath ledges in both coral and rubble habitats.

Fish collection. Settlement-stage Pomacentrus amboinensis were collected overnight using light traps moored at the back of the reef, and fish were transported to the Lizard Island Research Station at dawn. Juvenile $P$. amboinensis were collected from the base of shallow reefs approximately $1 \mathrm{wk}$ after settlement, using the anesthetic clove oil and hand nets. All fish were maintained in $25 \mathrm{l}$ flow-through aquaria systems for $24 \mathrm{~h}$ and fed ad libitum newly hatched Artemia sp. twice per day to facilitate recovery from the stress of capture. Growth during this period was minimal.
Adult Pseudochromis fuscus, Thalassoma lunare, Synodus dermatogenys and Cephalopholis microprion were collected from surrounding reefs using a combination of anesthetic (clove oil/sea water mix), hand nets, barrier nets and baited hand lines. Immediately following collection, all fish were maintained in individual 57L flow-through aquaria systems for $48 \mathrm{~h}$ before use in aquarium trials. Fish were not fed during this period to standardize for satiation, and to avoid handler-associated learning. This level of food deprivation is not thought to be unusual in the wild, given that available information suggests a high degree of gut emptiness for piscivores and generally slow through put rates through the digestive system (Sweatman 1984, Martin 1994).

Expt 1: Predator selectivity at time of settlement. Settlement-stage Pomacentrus amboinensis were placed into a clip-seal bag of aerated water and measured for standard length (SL $\pm 0.1 \mathrm{~mm}$ ) using calipers. Fish were then placed into one of 3 size classes: small (10.8 to $11.5 \mathrm{~mm} \mathrm{SL}$ ), medium (11.9 to $12.1 \mathrm{~mm} \mathrm{SL}$ ) and large (12.5 to $13.0 \mathrm{~mm} \mathrm{SL).} \mathrm{Size} \mathrm{classes} \mathrm{were} \mathrm{chosen} \mathrm{to}$ represent the entire range of sizes present at the time of settlement. Although the total range may be considered slightly conservative for this species, the size range present in individual light trap catches can vary considerably between days. The chosen classes allowed trials to be run over consecutive days whilst still maintaining the highest possible proportion of the total species' size range.

Predatory fish were also measured for SL prior to the beginning of trials. The size range of all 4 species used in trials was as follows: Pseudochromis fuscus, 40.0 to $71.4 \mathrm{~mm} \mathrm{SL}$; Thalassoma lunare, 52.0 to $167.6 \mathrm{~mm} \mathrm{SL}$; Synodus dermatogenys, 39.0 to $102.0 \mathrm{~mm} \mathrm{SL}$; and Cephalopholis microprion, 97.4 to $155.0 \mathrm{~mm}$ SL. Although larger individuals of $T$. lunare and $S$. dermatogenys were caught, they were not used in trials because of difficulties associated with acclimation in aquaria.

Eighteen identical flow-through aquaria were constructed, as per Almany et al. (2007). Each aquarium had an internal volume of $57.41(600 \times 255 \times 375 \mathrm{~mm})$. Aquaria were divided into 2 equal sized sections by a removable opaque Perspex partition. A $15 \mathrm{~cm}$ length of $105 \mathrm{~mm}$ diameter PVC pipe cut in half was placed into one section of the aquarium as the predator shelter. A single, artificial (white moulded resin) branching coral (item no. 21505; Wardleys/TFH; dimensions: $140 \times$ $115 \times 50 \mathrm{~mm}$ ) was placed in the other section as the prey shelter. Aquaria were surrounded by black plastic to visually isolate them from each other and other external disturbances. A small hole was cut in one side of the plastic to allow observation of the trials. All aquaria were maintained under natural lighting 
regimes (i.e. regular daylight hours), with experimental trials commencing between 10:00 h and 14:00 h of each day.

At the commencement of each trial, aquaria were divided in half with the opaque partition. Three settlement-stage Pomacentrus amboinensis (one from each of the 3 size classes) were placed into one half along with the artificial branching coral. The size difference of individuals between size classes within a trial was always at least $0.5 \mathrm{~mm}$. This corresponds to a body depth difference of approximately $0.31 \mathrm{~mm}$. Given that the mean body depth of individuals caught in light traps during the course of the experiment was $4.87 \mathrm{~mm}$, this difference of $0.31 \mathrm{~mm}$ represents $\sim 6.4 \%$ of the mean prey body depth. A single predator of known species and SL was allowed to acclimate in the opposite section of the aquaria for $48 \mathrm{~h}$ prior to the trials. Prey were acclimated for $1 \mathrm{~h}$ before the partition was removed and the trial started. Prey abundance was continuously monitored for the first $20 \mathrm{~min}$ and every $10 \mathrm{~min}$ thereafter. When one or more of the prey were found to be missing, the trial was ended. Any survivors were re-measured for SL to determine the identity of the missing individual(s). If all 3 prey were found to be missing, the trial was discarded. Forty successful trials were completed using Pseudochromis fuscus as the predator, 48 using Thalassoma lunare, 43 using Synodus dermatogenys, and 48 using Cephalopholis microprion. Predatory fish were each used in one trial only (whether successful or not) and were released at their point of capture when trials were completed.

Expt 2: Predator selectivity during early juvenile period. Juvenile Pomacentrus amboinensis were measured as per the previous experiment. Fish were subsequently placed into one of 5 size categories according to their SL: 11.8 to $13.0,13.1$ to $15.0,15.1$ to 17.0 , 17.1 to 19.0 , and 19.1 to $22.0 \mathrm{~mm}$. These classes were chosen so as to cover a size range from the time of settlement to approximately 3 to $4 \mathrm{wk}$ post-settlement. Two predator species only were used in trials with juvenile prey: Pseudochromis fuscus and Cephalopholis microprion. As in the previous experiment, predator SL was also measured, with the size range of the 2 species as follows: P. fuscus, 43 to $70 \mathrm{~mm} \mathrm{SL}$; and C. microprion, 79 to $145 \mathrm{~mm}$ SL.

The same experimental aquaria and protocol were used as in Expt 1, the only difference being that 5 prey (one from each of the 5 size classes) were placed into one half of the aquaria along with the artificial branching coral. The size difference between classes within a trial ranged from 1.4 to $3.3 \mathrm{~mm}$, representing $~ 8.7$ to $20.5 \%$ of the mean prey SL (16.1 mm). When 1 or more of the prey were found to be missing, the trial was ended. Any survivors were re-measured for SL to determine the identity of the missing individual(s). If more than 2 prey were missing, the trial was discarded. Sixty-nine successful trials were completed using Pseudochromis fuscus as the predator, whilst 45 were completed using Cephalopholis microprion.

Predatory gape limitation. Before predators were released, a measure of maximum gape size was taken for all 4 species, in order to obtain body length-gape size relationships. Maximum gape size was taken as the maximum internal horizontal distance within the fish's mouth without visible distortion. This was obtained by extending pincer calipers within the mouth (at the axial point between the upper and lower jaws) until the point of resistance.

Body depth and SL measurements were taken from a number of settlement-stage (collected from light traps) and juvenile (collected from surrounding reefs) Pomacentrus amboinensis to calculate body length-depth relationships. Body depth was obtained using calipers and taken as the widest vertical distance along the fish's length. Dorsal and ventral fins were not included in this measurement owing to their delicate and nonrigid nature in this species at this point in development. Both measurements were taken on the observation that prey were almost always ingested by the predator tail first and oriented on their side. Thus, any gape limitation will be driven by the maximum width of the predator's gape in comparison to the maximum body depth of the prey.

Body length:gape size ratios of the 4 predator species and body length:depth ratios of $P$. amboinensis were then compared to determine the extent to which predator-prey relationships were potentially limited by predator gape size.

Analysis. The number of times each size class was selected first was totaled for comparison within and between predator species and sizes for both experiments. In cases where 2 prey were taken before the trial was ended, each of the missing size classes was assigned a half count (0.5) and included in the analysis. Each of the predator species was further divided into 3 size classes to examine changes in size selection with ontogeny (Pseudochromis fuscus: 40 to 51, 52 to 60,61 to $70 \mathrm{~mm} \mathrm{SL;} \mathrm{Thalassoma} \mathrm{lunare:} 52$ to 88,89 to 110 , 111 to $160 \mathrm{~mm}$ SL; Synodus dermatogenys: 39 to 62, 63 to 79,80 to $102 \mathrm{~mm} \mathrm{SL;} \mathrm{Cephalopholis} \mathrm{microprion:} 79$ to 115,116 to 127,128 to $145 \mathrm{~mm} \mathrm{SL}$ ). The selection counts of the 3 prey size classes were compared for each predator species (total) and predator size class, using a chi-squared goodness of fit test. Selective profiles were compared between predator species and size class using a generalized linear model incorporating an ordinal multinomial distribution. This method was deemed the most appropriate form of analysis because of the categorical nature of the multinomial variables (size classes; 


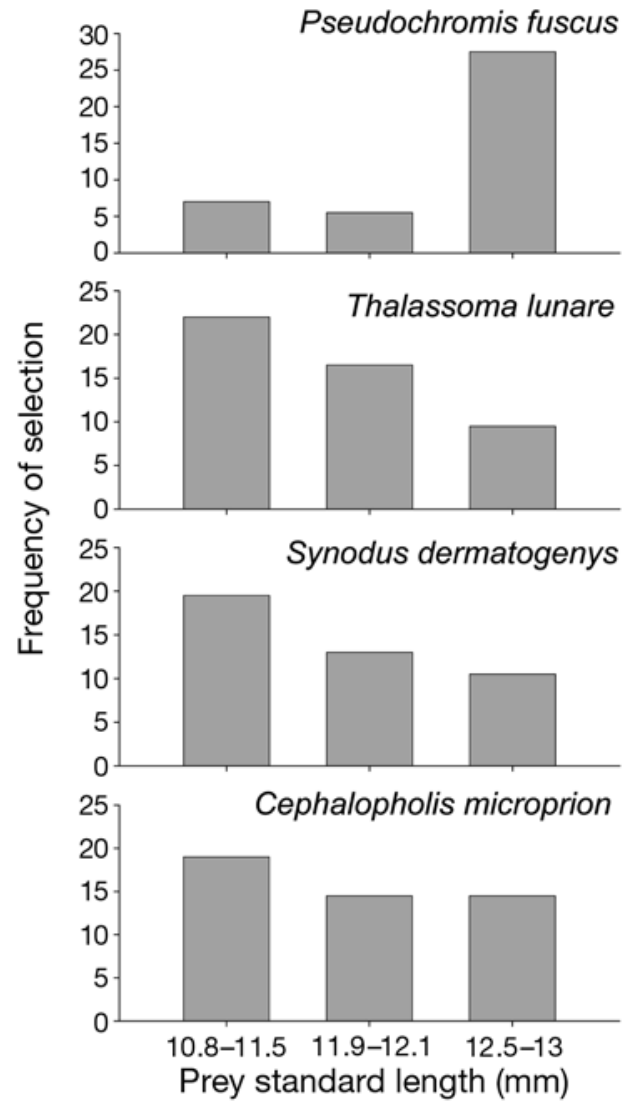

Fig. 1. Frequency of first selection of settlement stage Pomacentrus amboinensis during aquarium-based predation trials with 4 important predatory fish species

Perkins 2007). Assumptions of homogeneity of variance and normality were examined prior to analysis.

In order to determine the potential for gape limitation within interactions, predator body length-gape size relationships were plotted for each of the 4 species. This plot was then overlaid with the range of body depths of both the settlement-stage and juvenile prey individuals used in trials. The result allows for the visual comparison of predator sizes that are potentially constrained by maximum gape during interactions with settlement-stage and juvenile prey. Prey body length-depth relationships were then used in conjunction with predator body length-gape size relationships to estimate the number of times predators fed above their maximum gape during predation trials.

\section{RESULTS}

\section{Expt 1: Selectivity at settlement}

The size-selection profiles for settlement-stage Pomacentrus amboinensis differed significantly among the 4 predator species (Wald Statistic $; \chi_{\text {df } 3}^{2}=20.375, \mathrm{p}=$ 0.0001) (Fig. 1). Pseudochromis fuscus selected large prey $(68.75 \%$ of cases) significantly more often than small or medium prey $(17.50$ and $13.75 \%$ of cases respectively; $\chi_{\mathrm{df} 2}^{2}=22.663, \mathrm{p}<0.0001$ ). In contrast, both Thalassoma lunare and Synodus dermatogenys displayed a non-significant trend to select the smallest of the 3 size classes (47.83 and $45.35 \%$ of cases respectively) compared to the medium and large $P$. amboinensis (35.87 and $20.65 \%$, respectively, for T. lunare, $\chi_{\text {df } 2}^{2}=$ $4.906, \mathrm{p}=0.0860$; and 30.23 and $24.42 \%$, respectively, for $S$. dermatogenys, $\chi_{\text {df } 2}^{2}=0.656, p=0.8438$ ). Cephalopholis microprion showed no clear preference between prey sizes, with small, medium and large size classes being selected relatively evenly $\left(\chi^{2}\right.$ df $2=0.656$, $\mathrm{p}=0.8438)$.

There was no difference in size selection between predator sizes within all 4 of the predator species (Pseudochromis fuscus, Wald Statistic $\chi_{\text {df } 2}^{2}=0.368, \mathrm{p}=$ 0.832; Thalassoma lunare, Wald Statistic $\chi_{\text {df } 2}^{2}=1.037$, $\mathrm{p}=0.595 ;$ Synodus dermatogenys, Wald Statistic $\chi^{2}$ df 2 $=0.145, \mathrm{p}=0.93$; and Cephalopholis microprion, Wald Statistic $\chi_{\text {df } 2}^{2}=0.143, \mathrm{p}=0.931$ ).

\section{Expt 2: Selectivity during early juvenile stage}

There was a significant difference in the size-selective profiles of the 2 predator species during trials with early juvenile Pomacentrus amboinensis (Wald Statistic $\chi_{\text {df } 1}^{2}=17.764, \mathrm{p}<0.0001$ ) (Fig. 2). Pseudochromis fuscus selected the smallest prey size signifi-

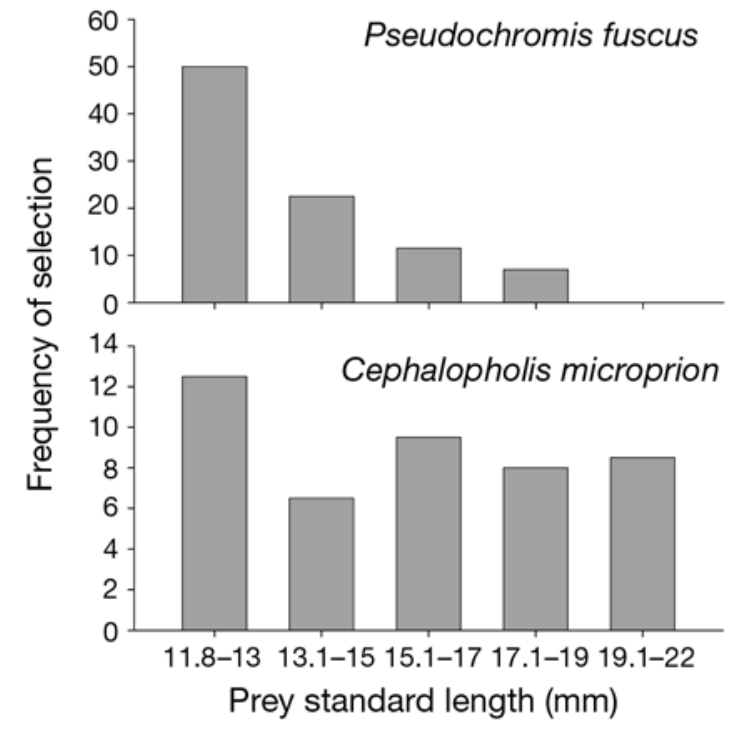

Fig. 2. Frequency of first selection of early juvenile Pomacentrus amboinensis during aquarium-based predation trials with 2 important predatory fish species 
cantly more often ( $54.95 \%$ of cases) than the 4 other size classes $(24.72,12.64,7.69$ and $0 \%$ of cases respectively; $\left.\chi_{\text {df } 4}^{2}=59.297, \mathrm{p}<0.0001\right)$. The largest size class (from 19 to $22 \mathrm{~mm}$ SL) was not selected by $P$. fuscus during any trials throughout the experiment. Interestingly, although the direction of selection changed between experiments, the size class targeted by $P$. fuscus in this experiment (smallest) roughly corresponds to the size range of the size class targeted in Expt 1 (largest). Cephalopholis microprion showed no detectable preference for prey size during interactions with juveniles $\left(\chi_{\mathrm{df} 4}^{2}=2.222, \mathrm{p}=0.6950\right)$, with the 5 size classes having a similar probability of selection.

Predator size had no effect on size-selective preference within species, with no significant difference being detected between the 3 size classes within both Pseudochromis fuscus (Wald Statistic $\chi_{\text {df } 2}^{2}=1.735, \mathrm{p}=$ 0.4200) and Cephalopholis microprion (Wald Statistic $\chi_{\text {df } 2}^{2}=1.494, \mathrm{p}=0.4740$ ).

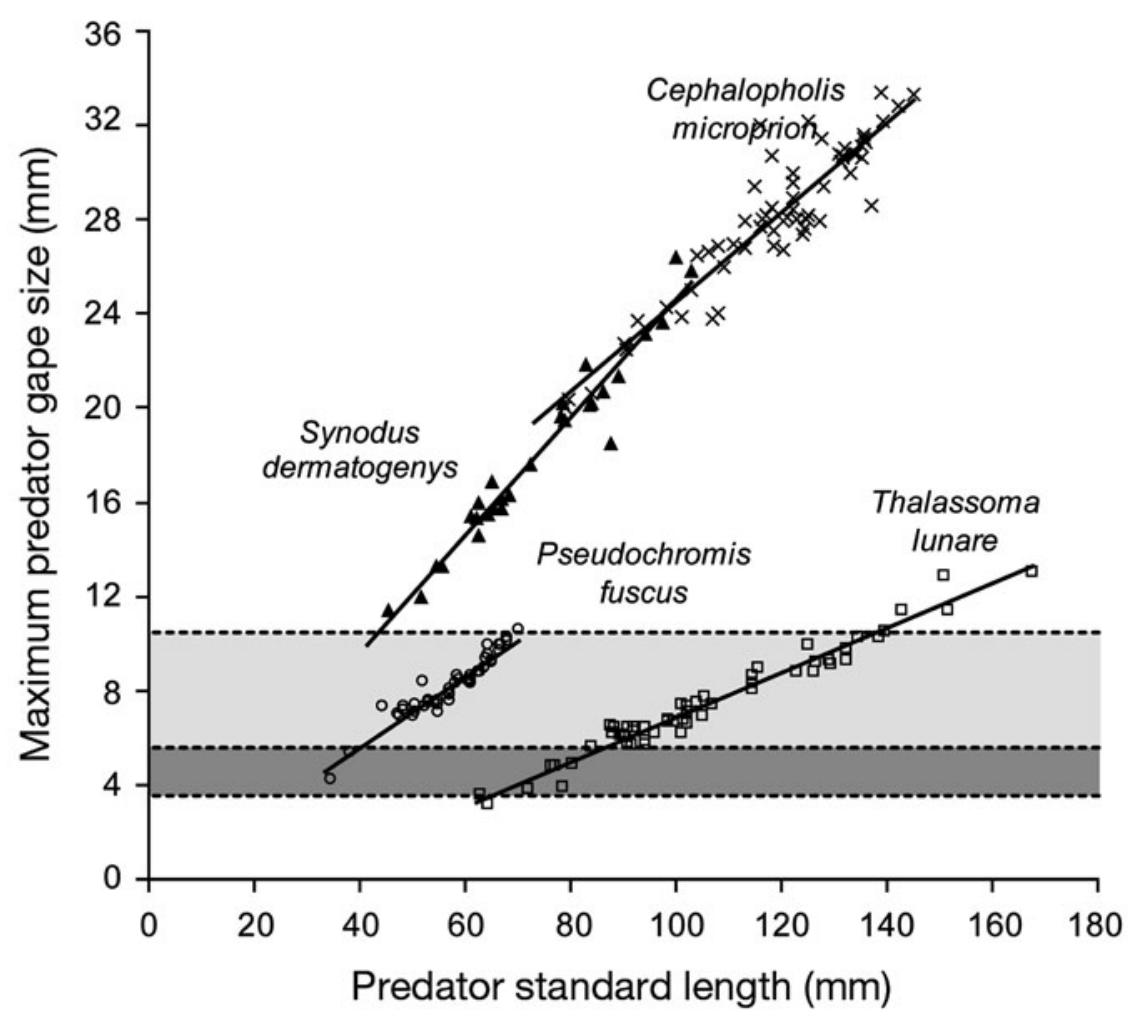

Fig. 3. Potential for gape limitation in 4 predator species (Pseudochromis fuscus, Thalassoma lunare, Synodus dermatogenys and Cephalopholis microprion) during interactions with settlement-stage and juvenile prey (Pomacentrus amboinensis). The range of prey body depths have been overlaid on the predator standard length-gape size relationships (dark and light grey: body-depth range of settlement-stage prey and juvenile prey, respectively) to ascertain the identity and size of those predators potentially constrained by gape size during predatory interactions (i.e. those individuals below or within the prey body-depth ranges)

\section{Predator gape limitation}

Prey body length-depth relationships were characterised by positive regressions for both settlementstage $\left(y=0.505 x-1.0761, R^{2}=0.819\right)$ and juvenile Pomacentrus amboinensis $\left(y=0.564 x-1.235, \mathrm{R}^{2}=\right.$ 0.963). Settlement-stage individuals had lower length and depth limits of 10.6 and $3.9 \mathrm{~mm}$ and upper limits of 13.0 and $5.7 \mathrm{~mm}$, respectively, whilst juveniles used in this study had lower length and depth limits of 11.8 and $5.1 \mathrm{~mm}$ and upper limits of 21.1 and $10.4 \mathrm{~mm}$, respectively.

Constraints imposed by predator gape size have the potential to greatly influence the outcome of interactions between both Pseudochromis fuscus and Thalassoma lunare, and juvenile/settlement-stage Pomacentrus amboinensis (Fig. 3). Given that the determinants of maximum prey size are predator gape width and prey body depth, the relationship suggests that all sizes of $P$. fuscus measured during this study were limited to varying extents by maximum gape during interactions with prey $>21 \mathrm{~mm}$ SL. As the maximum size at settlement for $P$. amboinensis is $\sim 13.3 \mathrm{~mm}$ SL, gape limitation will play a role in determining the capture probabilities of settlementstage $P$. amboinensis for any $P$. fuscus below $\sim 44 \mathrm{~mm}$ SL. In the case of $T$. lunare, any fish below $144 \mathrm{~mm}$ SL will potentially have limitations imposed by gape size during interactions with juvenile prey > $21 \mathrm{~mm}$ SL. Any T. lunare less than $91 \mathrm{~mm}$ SL may also be gape limited during interactions with settlement-stage prey.

Neither Synodus dermatogenys nor Cephalopholis microprion, of the sizes collected and measured in this study, appear to be constrained by maximum gape size during interactions with juvenile or settlement-stage Pomacentrus amboinensis (Fig. 3). Interpolation suggests that $S$. dermatogenys may potentially have limitations imposed at sizes below $47.7 \mathrm{~mm} \mathrm{SL}$, whilst $C$. microprion may be limited at sizes below $56.8 \mathrm{~mm}$ SL.

Using the calculated predator body length-gape size and prey body length-depth relationships as a guide (Fig. 3), it was determined that of the 99 trials in which Pseudochromis fuscus were provided a choice including prey sizes above their predicted maximum, individuals selected above this 
maximum on 8 occasions ( $8.1 \%$ of trials). In comparison, of the 20 trials in which Thalassoma lunare were offered a choice of prey including sizes above their predicted maximum, individuals selected above this maximum on 16 occasions ( $80 \%$ of trials). Measurement error was ruled out as a cause of this unexpected result because of the magnitude of the discrepancy, with prey body depth often exceeding maximum predator gape by up to 2.5 times. Owing to the proportionately larger gapes on both Synodus dermatogenys and Cephalopholis microprion, no trials were conducted on either species that involved a choice of prey above their predicted maximum. Of additional interest are the position and gradient of the body length-gape size relationship of $T$. lunare $(y=10.136 x+30.67)$ in comparison to that of $P$. fuscus, $S$. dermatogenys and $C$. microprion $(y=5.9923 x+8.2031, y=3.7987 x+5.2222$, and $y=4.4714 x-6.8009$, respectively), indicating that gape is significantly smaller for a given size in this species and that it increases with ontogeny at a slower rate than the others.

\section{DISCUSSION}

For juvenile coral reef fishes the direction and intensity of selection can differ over very small spatial scales (Holmes \& McCormick 2006, McCormick \& Meekan 2007, Samhouri et al. 2009). This variation is thought to be partially due to differences in the composition of predator assemblages between sites and their underlying selective preferences. However, this conclusion is based on inference due to the almost complete lack of information on species-specific predator selectivity within marine systems, and ecosystems in general (for exceptions, see Scharf et al. 2000, Allen 2008). This study presents one of the first cases of species-specific size selectivity for a number of important predators within an ecosystem. The results show that different predator species have different size-selective preferences during interactions with the settlement and early juvenile stages of a common coral reef fish species. While this gross finding of species differences matches the predictions of foraging theory, the details of the selection of prey by particular species does not conform well to predictions. Evidence suggests that at the time of settlement in the life of a coral reef fish, no particular body size confers a definitive survival advantage during predatory encounters. Instead, prey survival may in part be determined by the suite of predators present at the location of settlement, and how they interact with one another.

The idea that prey body size has a large influence on the outcome of predator-prey encounters is not new to the field of ecology (Litvak \& Leggett 1992, Janzen
1993, Mathis et al. 2003). The most common view amongst ecologists is that larger prey size conveys a universal survival advantage during such events (i.e. bigger is better; Rice et al. 1993, Takasuka et al. 2003, Wang et al. 2007). However, previous studies by Sogard (1997) and Allen (2008) have both found evidence to suggest otherwise, concluding that although larger prey size conveyed a survival advantage in a majority of species-specific interactions (negative size selection), there were a number of cases in which it was also selected against (positive size selection). Our study describes a similar pattern of mixed selective direction amongst a number of predator species on settlementstage prey, within a single system and under identical experimental conditions. Incidentally, a recent field study by McCormick \& Meekan (2007) also produced evidence to suggest similar patterns of contrasting selective preference in Thalassoma lunare and Pseudochromis fuscus. Although this outcome was largely implied, owing to difficulties associated with controlling external factors in field experiments, it shows that the results obtained from aquarium trials in this study hold true in a field context. This further reinforces the potential for interspecific differences in selective preference amongst predators and, in conjunction with our study, suggests that the role of prey body size in determining the outcome of predatory interactions should be considered on a case-by-case basis, rather than in general terms.

So why may a particular expression of the body length trait not provide a uniform survival advantage across all interactions, as predicted by 'bigger-is-better' theory? The answer to this is likely to lie within variation in the predation mode and morphology between predator species (Keast \& Webb 1966, Gaughan \& Potter 1997, Labropoulou \& Eleftheriou 1997). Such differences may potentially allow each predator species to exploit the available population in a different way, so as to optimize energy return. For example, the mobile and vigilant nature of Pseudochromis fuscus may enable it to choose and target optimally sized prey, whilst the opportunistic (Thalassoma lunare) and ambush nature (Synodus dermatogenys and Cephalopholis microprion) of predation by the other 3 species means that they may target anything that becomes vulnerable or comes within striking range. Such a suggestion has previously been made by Scharf et al. (2000), who concluded that size-based feeding strategies were related to predator foraging tactics, habitat overlap with prey, and morphological specializations that are particularly suited to specific habitats and/or prey types. Given the wide range of predator morphologies and feeding modes present amongst tropical reef fish communities (Hixon 1991), it is reasonable to suggest that the same may apply to this system. 
With the exception of Pseudochromis fuscus, and to a lesser extent Thalassoma lunare (only the smaller sizes are potentially constrained), gape size appears to play a minimal role in determining selective preferences amongst important predatory fish during interactions with newly settled and juvenile Pomacentrus amboinensis. In the case of $T$. lunare, the evidence against gape limitation during these relationships is further heightened by the apparent lack of constraint imposed by maximum gape size, with fish regularly recorded as feeding well above their predicted maximum prey size. This, however, is not necessarily surprising, given the opportunistic nature of T. lunare's predation mode and the observation that gape size of the species is relatively small for a given size and increases at a relatively slow rate with ontogeny. This observation indicates that gape size may not necessarily be a limiting factor in prey choice for this species. Alternately, this lack of constraint based on conventional morphological measurements could highlight the importance of other facets of jaw functional morphology (i.e. biomechanics) in influencing strike speed, jaw closure speed and bite force for this species (Grubich et al. 2008, Anderson \& Westneat 2009). Despite their being identified as important predators of juvenile reef fish, only the smallest sizes of Cephalopholis microprion and Synodus dermatogenys have the potential to be gape limited in this system. However, no individuals of this size were tested in this study, owing entirely to problems associated with locating and catching fish of such a small size for these species. The lack of gape limitation was particularly obvious in C. microprion, and this large gape size in comparison to the size range of the prey, as well as the 'engulfing' nature of their attacks, helps to explain the lack of selectivity observed for this species.

Despite its absence in the other 3 species, gape limitation was apparent in Pseudochromis fuscus during interactions with juvenile prey, with individuals rarely feeding above their predicted maximum size. However, during interactions with both settlement-stage and juvenile prey, fish were generally observed to feed well below their predicted maximum, indicating that the observed selective patterns were more a result of behaviour rather than morphological constraints. Overall, this general lack of a gape constraint for predators feeding on newly settled Pomacentrus amboinensis suggests that an important determinant of size selectivity during this period may be the behavioural traits exhibited by predator and prey (Gaughan \& Potter 1997).

Evidence for the involvement of prey behaviour in influencing susceptibility to predation has grown in recent years (Biro et al. 2004, 2006, Sih et al. 2004, Stamps 2007, Biro \& Stamps 2008). In the context of coral reef ecosystems, a recent field study by Meekan et al. (in press) found that Pomacentrus amboinensis of larger standard length spent more time foraging, were more aggressive towards smaller conspecifics, and swam greater distances than their smaller counterparts. These differences have also been shown to apply in an aquarium situation, with similar behavioural patterns occurring on the coral mould habitat used in this study (T. H. Holmes \& M. I. McCormick unpubl. data). It is possible that such differences in behaviour and space use between prey sizes may interact with predator feeding strategies and habitat niches to cause the observed selective patterns.

Despite our results, there is currently a significant body of literature suggesting that larger prey body size generally provides a survival advantage during the early post-settlement period in coral reef fishes (e.g. Schmitt \& Holbrook 1999, Searcy \& Sponaugle 2001). Where these studies differ from the current research is in the duration over which selection is measured, which tends to be days to weeks. This later assessment has the potential to mask selective forces acting within the first $24 \mathrm{~h}$ post-settlement, when individuals are most naïve to reef-based predators and hence most susceptible to predation. For those individuals that make it through this period, the probability of survival during future encounters has been shown to increase as a result of their experiences (McCormick \& Holmes 2006). This is presumably caused by behavioural changes within the newly settled individuals, which may in turn also affect the selective nature of predation.

The rapid change in the dynamics between predator and prey and its influence on prey selection have recently been highlighted by Meekan et al. (in press) in their study of behaviourally mediated mortality on open patch reefs. They found mortality to be selective towards larger individuals at the time of settlement, and towards smaller individuals a month following settlement. A similar pattern was observed in this study during trials with Pseudochromis fuscus, with prey selection acting against larger individuals at the time of settlement, and against smaller individuals during the early juvenile period. This selection occurred despite the choice of prey sizes largely falling within gape constraints. One explanation for this may be that this prey size (which was the same in both experiments, despite the different size range of classes around it) represents the optimal choice for this particular predator species, with each experiment detecting one end only of the resulting dome-shaped curve (Rice et al. 1993). An alternate explanation may lie within prey behaviour. At the upper end of this prey size spectrum, gape limitation will indeed play a role for this predator species. However, for the smaller prey sizes it is possible that experiences acquired by prey 
individuals during early settled life resulted in behavioural changes that make larger body size, and its associated covariates, distinctly advantageous. If such patterns are common, then it is important to distinguish between these 2 periods when assessing selective loss.

According to optimal foraging theory, preferred prey size should increase with increasing predator size (MacArthur \& Pianka 1966, Hughes 1980, Rice et al. 1993). However, this was not the case in our study, with predator body size failing to influence prey size selection for any of the predator species, during either the settlement or early juvenile trials. It may be that the size range of prey at the time of settlement was simply insufficient to cause a difference in selective preference, particularly in the case of the non gape-limited predators (i.e. Synodus dermatogenys and Cephalopholis microprion). During the juvenile trials, all sizes of Pseudochromis fuscus were largely constrained, by gape size, to the smaller size classes, limiting the potential for selective shifts, whilst the large gape size of C. microprion effectively negated any change. Alternately, the lack of change in prey size selection may be related to prey behaviour, in that specific prey sizes may be more vulnerable to predation by specific feeding strategies, which may not change greatly over the size range of predators used in this study (Bilcke et al. 2007, authors' unpubl. data). Whatever the underlying mechanisms, in relation to the predator species used in this study, predator body size appears to play a relatively minor role in determining the outcome of predatory interactions during early settled life.

The importance of selective processes in structuring community dynamics of organisms with complex life histories has received much attention in recent years (Congdon et al. 1999, Chivers et al. 2001, Searcy \& Sponaugle 2001, Allen 2008). Although caution should be observed when extrapolating these results to the wider prey community (because of the potential for morphological and behavioural differences between prey species), this study adds significantly to this literature and provides us with further insight into the patterns of predator-induced selectivity operating during a critical life stage for coral reef fishes. In extrapolating these results to the wider prey community, we have shown that there is great potential for such selective processes to differ between locations, on the basis of the composition of the predator community alone. In addition, this work highlights the importance of distinguishing between the settlement and early juvenile periods when assessing the mechanisms underlying selective loss. However, how these patterns hold in multi-species situations remains to be seen, owing to the possibility of synergistic and antagonistic relationships between predatory and other non-predatory species altering individual preferences (e.g. McCormick \&
Meekan 2007, Samhouri et al. 2009). Such factors must be considered before any assessment of selective patterns in natural systems is conducted.

Acknowledgements. We thank J. Scannell, M. Knott, P. Brading, A. Walsh, L. MacDonald and A. Villacorta Rath for their assistance in the field. Comments made by P. Mansell and S. Wilson on the manuscript were greatly appreciated. The staff of the Lizard Island Research Station (a facility of the Australian Museum) provided logistical support. This project was funded through grants supplied by the Australian Research Council Centre of Excellence for Coral Reef Studies, Lizard Island Doctoral Fellowship program, Ecological Society of Australia, and the Linnean Society of New South Wales. Research was conducted under the James Cook University animal ethics guidelines.

\section{LITERATURE CITED}

Allen JD (2008) Size-specific predation on marine invertebrate larvae. Biol Bull 214:42-49

Almany GR, Webster MS (2006) The predation gauntlet: early post-settlement mortality in coral-reef fishes. Coral Reefs 25:19-22

Almany GR, Peacock LF, Syms C, McCormick MI, Jones GP (2007) Predators target rare prey species in coral-reef fish assemblages. Oecologia 152:751-761

Anderson PSL, Westneat MW (2009) A biomechanical model of feeding kinematics for Dunkleosteus terrelli (Arthrodira, Placodermi). Paleobiology 35:251-269

Bilcke J, Herrel A, Aerts P (2007) Effect of prey- and predator size on the capture success of an aquatic snake. Belg J Zool 137:191-195

Biro PA, Stamps JA (2008) Are animal personality traits linked to life-history productivity? Trends Ecol Evol 23:361-368

Biro PA, Morton AE, Post JR, Parkinson EA (2004) Overwinter lipid depletion and mortality of age-0 rainbow trout (Oncorhynchus mykiss). Can J Fish Aquat Sci 61: 1513-1519

Biro PA, Abrahams MV, Post JR, Parkinson EA (2006) Behavioural trade-offs between growth and mortality explain evolution of submaximal growth rates. J Anim Ecol 75: 1165-1171

Brana F (2003) Morphological correlates of burst speed and field movement patterns: the behavioural adjustment of locomotion in wall lizards (Podarcis muralis). Biol J Linn Soc 80:135-146

Carr MH, Hixon MA (1995) Predation effects on early postsettlement survivorship of coral-reef fishes. Mar Ecol Prog Ser 124:31-42

- Chivers DP, Kiesecker JM, Marco A, Devito J, Anderson MT, Blaustein AR (2001) Predator-induced life history changes in amphibians: Egg predation induces hatching. Oikos 92: 135-142

Cohen JE, Pimm SL, Yodzis P, Saldana J (1993) Body sizes of animal predators and animal prey in food webs. J Anim Ecol 62:67-78

Congdon JD, Nagle RD, Dunham AE, Beck CW, Kinney OM, Yeomans SR (1999) The relationship of body size to survivorship of hatchling snapping turtles (Chelydra serpentina): an evaluation of the 'bigger is better' hypothesis. Oecologia 121:224-235

> Doherty PJ, Dufour V, Galzin R, Hixon MA, Meekan MG, Planes S (2004) High mortality during settlement is a pop- 
ulation bottleneck for a tropical surgeonfish. Ecology 85: $2422-2428$

Gaughan DJ, Potter IC (1997) Analysis of diet and feeding strategies within an assemblage of estuarine larval fish and an objective assessment of dietary niche overlap. Fish Bull 97:722-731

Grubich JR, Rice AN, Westneat MW (2008) Functional morphology of bite mechanics in the great barracuda (Sphyraena barracuda). Zoology 111:16-29

Hill JE, Nico LG, Cichra CE, Gilbert CR (2005) Prey vulnerability to peacock cichlids and largemouth bass based on predator-gape and prey body depth. Proc Annu Conf Southeastern Assoc Fish Wild Agencies 58:47-56

Hixon MA (1991) Predation as a process structuring coral reef fish communities. In: Sale PF (ed) The ecology of fishes on coral reefs. Academic Press, San Diego, CA, p 475-507

Holbrook SJ, Schmitt RJ (2002) Competition for shelter space causes density-dependent predation mortality in damselfishes. Ecology 83:2855-2868

$>$ Holmes TH, McCormick MI (2006) Location influences sizeselective predation on newly settled reef fish. Mar Ecol Prog Ser 317:203-209

Hughes RN (1980) Optimal foraging theory in the marine context. Oceanogr Mar Biol 18:423-481

$>$ Husseman JS, Muray DL, Power G, Mack C, Wenger CR, Quigley H (2003) Assessing differential prey selection patterns between two sympatric large carnivores. Oikos 101: 591-601

Janzen FJ (1993) An experimental analysis of natural selection on body size of hatching turtles. Ecology 74:332-341

Keast A, Webb D (1966) Mouth and body form relative to feeding ecology in the fish fauna of a small lake, Lake Opinicon, Ontario. Can J Fish Aquat Sci 23:1845-1874

Kerrigan BA (1996) Temporal patterns in size and condition at settlement in two tropical reef fishes (Pomacentridae: Pomacentrus amboinensis and P. nagasakiensis). Mar Ecol Prog Ser 135:27-41

Labropoulou M, Eleftheriou A (1997) The foraging ecology of two pairs of congeneric demersal fish species: importance of morphological characteristics in prey selection. J Fish Biol 50:324-340

Litvak MK, Leggett WC (1992) Age and size-selective predation on larval fishes: the bigger-is-better hypothesis revisited. Mar Ecol Prog Ser 81:13-24

MacArthur RH, Pianka ER (1966) On optimal use of a patchy environment. Am Nat 100:603-609

Martin J (1994) Predation on juvenile coral-reef fish at Lizard Island, northern Great Barrier Reef: an ecological and behavioural study. BSc (Hons) dissertation. James Cook University, Townsville

Mathis A, Murray KL, Hickman CR (2003) Do experience and body size play a role in responses of larval ringed Salamanders, Ambystoma annulatum, to predator Kairomones? Laboratory and field assays. Ethology 109: $159-170$

McCormick MI, Holmes TH (2006) Prey experience of predation influences mortality rates at settlement in a coral reef fish, Pomacentrus amboinensis. J Fish Biol 68:969-974

McCormick MI, Meekan MG (2007) Social facilitation of selective mortality. Ecology 88:1562-1570

McCormick MI, Makey L, Dufour V (2002) Comparative study of metamorphosis in tropical reef fishes. Mar Biol 141: 841-853

Meekan MG, von Kuerthy C, McCormick MI (in press) Does behaviour mediate the costs and benefits of fast growth in a marine fish? Anim Behav

Milicich MJ, Doherty PJ (1994) Larval supply of coral reef fish populations: magnitude and synchrony of replenishment to Lizard Island, Great Barrier Reef. Mar Ecol Prog Ser 110:121-134

Penteriani V, Del Mar Delagado M, Bartolommei P, Maggio C, Alonso-Alvarez C, Holloway GJ (2008) Owls and rabbits: predation against substandard individuals of an easy prey. J Avian Biol 39:215-221

Perkins SM (2007) Statistical inference on categorical variables. Methods Mol Biol 404:73-88

Persson A, Andersson J, Wahlstrom E, Eklov P (1996) Sizespecific interactions in lake systems: predator gape limitation and prey growth rate and mortality. Ecology 77: 900-911

Poling KR, Fuiman LA (1997) Sensory development and concurrent behavioural changes in Atlantic croaker larvae. J Fish Biol 51:402-421

Rice JA, Crowder LB, Rose KA (1993) Interactions between size-structured predator and prey populations: experimental test and model comparison. Trans Am Fish Soc 122:481-491

Risbey DA, Calver MC, Short J, Bradley JS, Wright IW (2000) The impact of cats and foxes on the small vertebrate fauna of Heirisson Prong, Western Australia. II. A field experiment. Wildl Res 27:223-235

Sakamoto M, Hanazato T (2008) Antennule shape and body size of Bosmina: key factors determining its vulnerability to predacious Copepoda. Limnology 9:27-34

Samhouri JF, Steele MA, Forrester GE (2009) Inter-cohort competition drives density dependence and selective mortality in a marine fish. Ecology 90:1009-1020

Scharf FS, Juanes F, Rountree RA (2000) Predator size-prey size relationships of marine fish predators: interspecific variation and effects of ontogeny and body size on trophicniche breadth. Mar Ecol Prog Ser 208:229-248

Schmitt RJ, Holbrook SJ (1999) Mortality of juvenile damselfish: implications for assessing processes that determine abundance. Ecology 80:35-50

Searcy SP, Sponaugle S (2001) Selective mortality during the larval-juvenile transition in two coral reef fishes. Ecology 82:2452-2470

> Sih A, Crowley P, McPeek M, Petranka J, Strohmeier K (1985) Predation, competition, and prey communities: a review of field experiments. Annu Rev Ecol Syst 16:269-311

Sih A, Bell AM, Johnson JC (2004) Behavioural syndromes: an ecological and evolutionary overview. Trends Ecol Evol 19:372-378

Slaughter JE, Jacobson B (2008) Gape: body size relationship of flathead catfish. N Am J Fish Manage 28:198-202

Sogard SM (1997) Size-selective mortality in the juvenile stage of teleost fishes: a review. Bull Mar Sci 60:1129-1157

Stamps JA (2007) Growth-mortality tradeoffs and 'personality traits' in animals. Ecol Lett 10:355-363

Stuart-Smith J, Swain R, Wapstra E (2007) The role of body size in competition and mate choice in an agamid with female-biased size dimorphism. Behaviour 144:1087-1102

Sweatman HPA (1984) A field study of the predatory behaviour and feeding rate of a piscivorous coral reef fish, the Lizardfish Synodus englemani. Copeia 1984:187-194

- Takasuka A, Aoki I, Mitani I (2003) Evidence of growth selective predation on larval Japanese anchovy Engraulis japonicus in Sagami Bay. Mar Ecol Prog Ser 252:223-238

Urban MC (2007) The growth-predation risk trade-off under a growing gape-limited predation threat. Ecology 88: $2587-2597$

Wang Y, Guo Z, Pearl CA, Li Y (2007) Body size affects the predatory interactions between introduced American bullfrogs (Rana catesbeiana) and native anurans in China: 
an experimental study. J Herpetol 41:514-520

Wellborn GA (1994) Size-biased predation and prey life histories: a comparative study of freshwater amphipod populations. Ecology 75:2104-2117

Werner EE (1974) The fish size, prey size, handling time relation, in several sunfishes and some implications. J Fish Res Board Can 31:1531-1536

Werner EE, Gilliam JF (1984) The ontogenetic niche and spe-

Editorial responsibility: Nicholas Tolimieri,

Seattle, Washington, USA cies interactions in size-structured populations. Annu Rev Ecol Syst 15:393-425

Wilbur HM, Morin PJ, Harris RN (1983) Salamander predation and the structure of experimental communities: anuran responses. Ecology 64:1423-1429

Woodward G, Ebenman B, Emmerson M, Motoya JM, Olesen JM, Valido A, Warren PH (2005) Body size in ecological networks. Trends Ecol Evol 20:402-409

Submitted: July 14, 2009; Accepted: September 25, 2009

Proofs received from author(s): December 12, 2009 